\title{
Circadian disruption in shift workers - the effects of insufficient sleep on dietary and lifestyle behaviours
}

\author{
F. Nea ${ }^{1}$, K. Poushahidi ${ }^{2}$, C. Corish ${ }^{1}$, J. Kearney $^{1}$, B. Livingstone ${ }^{2}$ and C. Bassul ${ }^{1}$ \\ ${ }^{1}$ Dublin Institute of Technology, Kevin Street, Dublin 8, Republic of Ireland and ${ }^{2}$ Northern Ireland Centre for Food and \\ Health, Centre for Molecular Biosciences, University of Ulster, Coleraine BT52 1SA, UK
}

\section{This abstract was awarded the student prize for best poster original communication}

Shift workers report more sleep problems compared to the general public, and it has been estimated that $10-30 \%$ of shift workers suffer from a circadian rhythm disorder known as "shift work disorder"(1). This quantitative study investigated the impact of shift work on the diet and lifestyle of workers $(n$ 1300) on the island of Ireland via telephone interview by a market research company. Subjects were males and females aged 18-64 years, who were employed in shift work at the time of survey. Questions were based on work patterns and dietary and lifestyle behaviours in the previous month, with all data being self-reported. Self-reported sleep duration was coded to assess compliance with the recommended duration of sleep for age, as outlined by the National Sleep Foundation $^{(2)}$. In the case of this sample, the ideal duration of sleep for those aged 18-64 years is 7-9 hours per 24 hours.

Overall, just $34.3 \%$ of the sample was achieving adequate sleep. A number of factors were associated with insufficient sleep - being male $(\mathrm{p}<0.001)$, being 35-54 years of age $(\mathrm{p}<0.001)$, having adult/child dependents $(\mathrm{p}<0.001)$, working in larger organisations $(\mathrm{p}=$ $0.045)$, working in distribution/logistics, manufacturing or construction $(p=0.005)$, working night shifts $(p=0.042)$, and working longer shifts $(p=0.002)$. Insufficient sleep had an effect on the diet of workers. Those who did not achieve adequate sleep were more likely to skip meals on working days and skipped meals significantly more frequently $(\mathrm{p}=0.023)$. Workers with insufficient sleep were also significantly less likely to consume the recommended 5 portions of fruit and vegetables per day $(37.5 \%$ vs $43.3 \%$, $\mathrm{p}=0.045)$ and were less likely to consume the recommended 3 portions of milk/cheese/yoghurt per day $(11.6 \%$ vs $8.1 \%$, p = $0 \cdot 050)$. In addition, those with insufficient sleep had higher prevalence of hypertension $(10.2 \%$ vs $5.7 \%, p=0.008)$ and depression/ anxiety $(7.3 \%$ vs $3.4 \%, p=0.008)$. Participants were also questioned how they perceived shift work impacts on various aspects of their lives. Compared to those who achieve adequate sleep, those who had insufficient sleep were significantly more likely to report that shift work had a negative effect on their physical health $(p<0.001)$, mental health $(p=0.003)$, family life $(p=0.001)$, social life $(p=0.046)$, physical activity levels $(p=0.029)$ and overall quality of life $(p=0.002)$. Those with insufficient sleep were also significantly more likely to report that shift work increases how much alcohol they drink $(p=0.041)$.

Shift work is negatively impacting on the sleep patterns of workers with a knock-on effect on dietary and lifestyle behaviours. Efforts are required to minimise the impact of circadian disruption by careful consideration of shift scheduling and targeted advice to shift workers.

1. Gumenyuk V, Roth T, Drake CL (2012) Circadian phase, sleepiness, and light exposure assessment in night workers with and without shift work disorder. Chronobiol Int 29(7), 928-36.

2. Hirshkowitz M, Whiton K, Albert SM, et al. (2015) National Sleep Foundation's sleep time duration recommendations: methodology and results summary. Sleep Health 1(1): 40-3. 